\title{
Selección de proveedores de insumos críticos en términos de sostenibilidad, a través de la metodología multicriterio, en una empresa del sector azucarero *
}

\author{
Hugo Ferney Ocampo-Murillo \\ Escuela de Ingeniería Industrial, Universidad del Valle, Cali - Colombia \\ hfocampo@gmail.com (I) https://orcid.org/0000-0002-9045-2689 \\ Martha Lucía Quintero-Garzón \\ Profesora Escuela de Ingeniería Industrial, Universidad del Valle, Cali - Colombia \\ martha.quintero@correounivalle.edu.co (D) https://orcid.org/0000-0002-8069-019X
}

RES U M N

Se presentan aquí, los resultados de una investigación teórico-práctica, en la cual se abarcó el eslabón de suministro, en una cadena de abastecimiento, en una empresa del sector azucarero en el Valle del Cauca. Para la selección de proveedores de insumos críticos, se aplicó la metodología multicriterio, considerando criterios que iban más allá del costo, tomando como referencia una clasificación de insumos de acuerdo a su criticidad en términos de sostenibilidad, para el proceso productivo. Inicialmente, se hizo una caracterización y una selección de los insumos críticos, en términos de sostenibilidad. Igualmente, se identificaron los proveedores para los insumos seleccionados. Luego, y en consenso con un comité escogido al interior de la empresa, se seleccionó y aplicó el método de jerarquía analítica AHP (Analytic Hierarcgy Process). Finalmente, se tomó la decisión más acorde a los requerimientos reales de la organización, los cuales consideraban como criterio principal la sostenibilidad, para alcanzar la competitividad y el desarrollo sostenible. Esto contribuirá con el tiempo, a que la organización sea económicamente viable, ambientalmente soportable y socialmente equitativa.

Palabras clave

Cadena de abastecimiento; sostenibilidad; insumo; proveedor; análisis de decisión multicriterio; selección

Clasificación JEL

\section{Suppliers' selection of critical supplies in terms of sustainability, through the use of multi-criteria methodology, in sugar sector Company}

A B S T R A C T

We undertake a theoretical-practical investigation in which we study the supply function carried out by a company in the sugar sector at the Valle del Cauca. For the selection of critical input suppliers, we apply a multi-criteria methodology in which we take into account other criteria besides cost by considering a supplies' classification based on how critical these supplies are for the production process in

Recibido: 05/06/2019 Aceptado: 10/01/2020

* El presente artículo es de naturaleza investigativa y no necesitó ningún tipo de financiación, realizado como caso de estudio aplicado en una empresa. En él se realiza la aplicación de proceso analítico jerárquico para la selección de proveedores de insumos críticos de acuerdo con la sostenibilidad en una empresa del sector azucarero. (http://creativecommons.org/licenses/by-nc-sa/4.0/) Published by Universidad Libre - Cali, Colombia.

Cómo citar este artículo: OCAMPO-MURILLO, Hugo Ferney; QUINTERO-GARZÓN, Martha Lucía. Selección de proveedores de insumos críticos en términos de sostenibilidad, a través de la metodología multicriterio, en una empresa del sector azucarero. En: Entramado. Julio - Diciembre, 2020. vol. I6, no. 2, p. 24-44. https://doi.org// 0.1804I//900-3803/entramado.2.6436 
terms of sustainability. Initially, we classify and select supplies that are more critical in regards to sustainability. Likewise, the suppliers for the selected inputs were identified. Then, in agreement with a committee chosen within the company, we select and apply the analytical hierarchy method AHP (Analytic Hierarchy Process). Finally, according to the real requirements of the organization, the most appropriate decision is made, which considers sustainability as the main criterion to achievecompetitiveness and sustainable development. This will contribute to an economically feasible, environmentally bearable, and socially equitable company.

KEYWORDS

Supply chain; sustainability; supplies, supplier; multicriteria decision analysis; selection

Jel Classification

\section{Selecção de fornecedores de inputs críticos em termos de sustentabilidade, através da metodologia multicritério, numa empresa do sector do açúcar}

RES U M O

São aqui apresentados os resultados de uma investigação teórico-prática, que abrangeu o elo de abastecimento de uma cadeia de abastecimento de uma empresa do sector do açúcar no Valle del Cauca. Para a selecção de fornecedores de inputs críticos foi aplicada a metodologia multicritérios, considerando critérios que iam além do custo, tomando como referência uma classificação dos inputs de acordo com a sua criticidade em termos de sustentabilidade, para o processo produtivo. Inicialmente, foi feita uma caracterização e selecção de inputs críticos em termos de sustentabilidade. Do mesmo modo, foram identificados os fornecedores dos factores de produção seleccionados. Em seguida, e em consenso com um comité escolhido na empresa, foi seleccionado e aplicado o método AHP (Analytic Hierarchy Process). Finalmente, foi tomada a decisão mais consentânea com os requisitos reais da organização, que considerou a sustentabilidade como o principal critério para alcançar a competitividade e o desenvolvimento sustentável. Isto contribuirá, com o tempo, para tornar a organização economicamente viável, ambientalmente sustentável e socialmente equitativa.

Palavras-chave Cadeia de abastecimento; sustentabilidade; input; fornecedor; análise de decisão multicritério; selecção

Classificação JEL

\section{Introducción}

Actualmente, la globalización y la responsabilidad social empresarial (RSE), han impulsado a las organizaciones, en su camino hacia el crecimiento y la competitividad. Para ello, deben gestionar sus cadenas de abastecimiento con una mirada global, que permita optimizar los recursos y ofrecer productos de calidad, que atiendan de forma rápida los requerimientos de los clientes en el mercado.

Dentro de la gestión de las cadenas de abastecimiento, un proceso importante es el abastecimiento de materias primas y/o insumos, el cual es necesario para realizar el proceso misional y operacional de toda compañía. Por ende, es de vital importancia contar con insumos que cumplan con los más altos estándares, para finalmente garantizar productos en esa misma clasificación.

Para llevar a cabo lo anterior, los encargados del aprovisionamiento en las compañías deben buscar y seleccionar proveedores que garanticen una serie de características que dependerán de las intenciones propias de las compañías en el marco de su objetivo misional; por lo que se hace indispensable o relevante contar con métodos de selección de proveedores claros y definidos que permitan tener control sobre el proceso inicial del aprovisionamiento.

De acuerdo a Barreneche (2010), la selección de proveedores es una actividad que realizan todas las 
empresas, dado que, para poner en marcha sus procesos necesitan de una serie de insumos los cuales impactaran directamente en el producto final y sus condiciones de calidad. Por tal motivo, la selección de proveedores óptimos puede traducirse en productos finales que garanticen una alta competitividad de las empresas.

La selección de proveedores hace parte del proceso de abastecimiento en las cadenas de suministro y su adecuada gestión podrá traer grandes beneficios para las compañías en su rendimiento global; lo anterior lo reflejan autores como Chopra y Meindl (2008) y Ballou (2004), quienes coinciden en que la efectividad de las cadenas de abastecimiento, radica en su adecuada gestión en todos los procesos y actividades correspondientes para cada uno de los eslabones.

Adicionalmente, de acuerdo con lo expuesto por González y Garza (2003), seleccionar un proveedor es un problema de decisión de múltiples atributos, el cual se resuelve normalmente de forma empírica. Sin embargo, en la actualidad con los robustos sistemas de calidad de las organizaciones, implementan técnicas matemáticas en la toma de decisiones que se convierten en una herramienta eficaz para la adecuada gestión.

Por lo anterior, y teniendo en cuenta, que la organización donde se realizó esta investigación, tomaba las decisiones de selección de proveedores basada en métodos empíricos, se propuso como objetivo, seleccionarlos proveedores de los insumos críticos en términos de sostenibilidad, usando una metodología multicriterio, que permitiera guiar a la empresa hacia una mejora significativa en los procesos de gestión y calidad, que le permitieran ser más competitiva en el sector azucarero.

Después de una revisión bibliográfica, se socializaron las diferentes técnicas consultadas con el comité escogido de la compañía y se decidió que la herramienta más práctica para este caso, era una de las técnicas multicriterio discretas, más conocida como proceso de jerarquía analítica $(A H P$ -
Analytic Hierarcgy Process), por su fácil entendimiento y aplicación para la organización. Esto debido a la explicación y motivación, que se hizo, en el manejo de la información, de tal forma, que el proceso de implementación de esta metodología, pareciera lo más amigable posible, para el personal involucrado.

Para complementar el artículo, se presenta a continuación un marco teórico que sustenta las temáticas tratadas en la investigación; igualmente, se platea una metodología, por medio de la cual, se lograron los objetivos propuestos; se presentan los resultados y una discusión de los mismos, al igual que unas conclusiones y las referencias bibliográficas.

\section{Marco teórico}

Según, Sarache, W., Castrillón, Ó., \& Ortiz, L. (2009), la selección de proveedores es una decisión de múltiples criterios y de impacto estratégico en las organizaciones. Los mismos autores muestran que el paradigma del múltiple criterio se presenta como una ayuda efectiva en la práctica de la toma de decisiones y gestión de las organizaciones; por lo anterior la selección de una herramienta multicriterio es de gran ayuda en la selección de proveedores para las empresas.

Por ello, es importante conocer no sólo la conceptualización de algunos términos, sino la relación y su cohesión para la selección de los proveedores, en una cadena de suministros.

\section{I. Gestión de la cadena de suministro}

La cadena de suministro es definida como el conjunto de proveedores y clientes que se encuentran conectados en sentido directo desde el proveedor inicial hasta el cliente final; de acuerdo a esto, la cadena de suministro o supply chain (SC) abarca todas las actividades asociadas con el flujo y transformación de bienes e información, desde la fase de materias primas hasta el usuario final. En la Figura I, se puede ver un claro ejemplo de los eslabones que se presentan en una cadena de suministro tradicional.

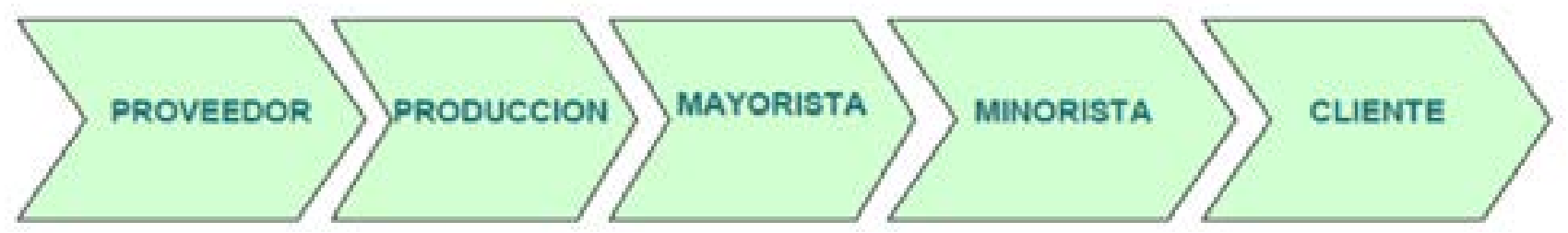

Figura I. Cadena de suministro tradicional Fuente: Vilana $(20 \mid 0)$. 
La administración de la cadena de suministro o SCM (Supply chain management), considera la adecuada gestión de recursos para la obtención resultados óptimos al momento de generar procesos y entregar el producto final, para ello se requiere que el análisis sea integrado, evitando errores de estudiar la cadena de suministro de una empresa como eslabones que no presentan relación. Por ende, se afirma que la administración de la cadena de suministro consiste en la integración de actividades a través de relaciones mejoradas que permitan adquirir una ventaja competitiva sostenible, mediante la gestión de sistemas de información, aprovisionamiento y compras, programación de la producción, tramitación de pedidos, gestión de inventarios, almacenamiento, servicio al cliente y servicio posventa (Vilana, 20I0).

Cabe resaltar que la gestión de la cadena de suministro presenta dos aspectos fundamentales: el primero, consiste en una gestión de la cadena interna, la cual administra los procesos entre los departamentos de una organización única; el segundo, consiste en administración de las relaciones de una organización con proveedores y clientes. Este segundo aspecto mencionado, presenta un enfoque holístico que permite ver la cadena de suministro como como una red de empresas y actividades que lleva a cabo las funciones de desarrollo de productos, obtención de materiales de los proveedores, movimiento de materiales entre instalaciones, producción de productos, distribución de bienes terminados a los clientes y servicio posventa.

La gestión de la cadena de suministro se compone de tres elementos:

- Procesos de negocio: son aquellos que pueden atravesar las fronteras intra e ínter organizacional, independientemente de la estructura formal. Son siete: gestión de la relación con el cliente,gestión del servicio al cliente, gestión de la demanda, cumplimiento del pedido, gestión del flujo de producción, aprovisionamiento, desarrollo de producto y comercialización.

- Componentes de gestión: hace referencia a componentes de la gestión que son comunes a todos los procesos de negocio y miembros de la cadena de suministro; donde se hace importante la gestión de estos componentes comunes, debido a que ellos determinan cómo son gestionados y estructurados los procesos de negocio $y$, por tanto, la cadena de suministro.

- Estructura: esta debe estar definida de acuerdo a consideraciones como complejidad del producto, número de proveedores disponibles y disponibilidad de materias primas. Las dimensiones a considerar incluyen la longitud de la cadena de suministro $y$, el número de proveedores y clientes. La relación más apropiada será aquella que mejor se ajuste al conjunto de circunstancias específicas.

Para que la gestión se lleve a cabo de la mejor manera posible, se necesita que la cadena de suministros, sea sostenible. A continuación, se presenta esa relación.

\section{Sostenibilidad de la cadena de suministro}

La sostenibilidad de una cadena de suministro se refiere a la coordinación e integración de procesos interorganizacionales entre los miembros de la cadena para cumplir sus objetivos sociales, medioambientales y económicos. Una cadena de suministro sostenible se define como la integración entre la gestión de materiales, información y flujos de capital considerando las dimensiones económicas, sociales y ecológicas.Al momento de hablar de sostenibilidad en la cadena de suministro debe considerarse la participación de los diferentes eslabones que la conforman como son: proveedores, productores, operadores logísticos y otros relacionados (Riveros y Rodríguez, 2016).

Las ideas principales al referirse a una cadena de abastecimiento sostenible son el asegurar la viabilidad a largo plazo y la continuidad del negocio, buscando un mejor uso de los recursos y logrando así impactar no solo el aspecto económico sino también el ambiental y el social. Se afirma entonces, que una cadena de suministro sostenible se da entre el aprovechamiento y control de los recursos y, la vigilancia y trazabilidad de todo el proceso (Ávila, 20I4).

La sostenibilidad debe involucrar diferentes factores como lo son: los participantes de la cadena de abastecimiento, las instituciones, el contexto comercial, y los aspectos sociales e institucionales. Adicionalmente, la sostenibilidad económica se encuentra asociada al desarrollo económico y sostenible, el cual permite afrontar las dificultades del presente sin poner en peligro las generaciones futuras (Castillo, 2011).

Con el fin de lograr una cadena de suministro sostenible se considera la estrategia de gerencia de proveedores, lo cual permite asegurar la calidad e integridad del bien 0 servicio final, mitigar riesgos e incrementar el rendimiento. Cabe resaltar la importancia de los proveedores al relacionarse con el concepto de sostenibilidad, debido a que el lograr sinergia con éstos, representa una fuente alta de competitividad, debido a que la empresa depende de sus propias operaciones y para determinadas extensiones en las operaciones de sus miembros la cadena de suministro, es decir, sus proveedores (Ávila, 2014). 
Una adecuada gestión de proveedores para una cadena de abastecimiento sostenible significa cambios positivos en los aspectos: ingresos, marca, costos y riesgos (Hanifan, Sharma, \& Mehta, 2012).

En la Figura 2, se observa una cadena de suministros sostenible.

Igualmente, existen unos retos que se encuentran relacionados con el concepto de una cadena de abastecimiento sostenible. Éstos se categorizan en tres: estratégico, regulatorio $y$ financiero.

Reto estratégico: se busca que el interés de los proveedores se encuentre acoplado con las expectativas de los stakeholders.

Reto regulatorio: consiste en lograr que la información requerida en consultas o generación de informes se encuentre disponible en el momento exacto en el que sea solicitada.

Reto financiero: aplicar operaciones estratégicas que permitan la reducción de costos y otros indicadores académicos.
Los beneficios que permite la cadena en cuestión son el aumentar la confianza con los proveedores, mejorar el posicionamiento de la empresa ante los inversionistas, incentivar a los trabajadores a proponer nuevas técnicas de innovación, ahorrar recursos y reducir residuos.

Para lograr una cadena de abastecimiento sostenible, las empresas deben seguir una serie de pasos entre los cuales se encuentran: realizar el mapa de su cadena de suministro, comunicar las expectativas, evaluar el rendimiento de los proveedores, desarrollar programas de capacitación, conducir la mejora del rendimiento y unirse a la colaboración de la industria (Ávila, 2014).

\subsection{Inventarios de insumos}

Los inventarios son definidos como un activo de la compañía y son un aspecto importante de la cadena de suministro, debido a que afectan directamente el nivel de respuesta y la eficiencia de la cadena (Vidal, 2004).

Los inventarios de insumos son aquellos en los que se contabilizan los materiales que no han sido modificados por el proceso productivo de las empresas. Para esta clase de

\begin{tabular}{|l|}
\multicolumn{1}{|c|}{ INGRESOS } \\
- La creación de nuevos modelos de \\
negocio \\
- Colaboración para desarrollar nuevos \\
mercados \\
- Innovación para desarrollar nuevos \\
productos y servicios
\end{tabular}

Seguro/ Corto plazo

\section{REDUCCIÓN DE COSTOS}

- Mejorar la eficacia energética

- Racionalización de la cadena de suministro y la logistica - Innovando con proveedores y clientes

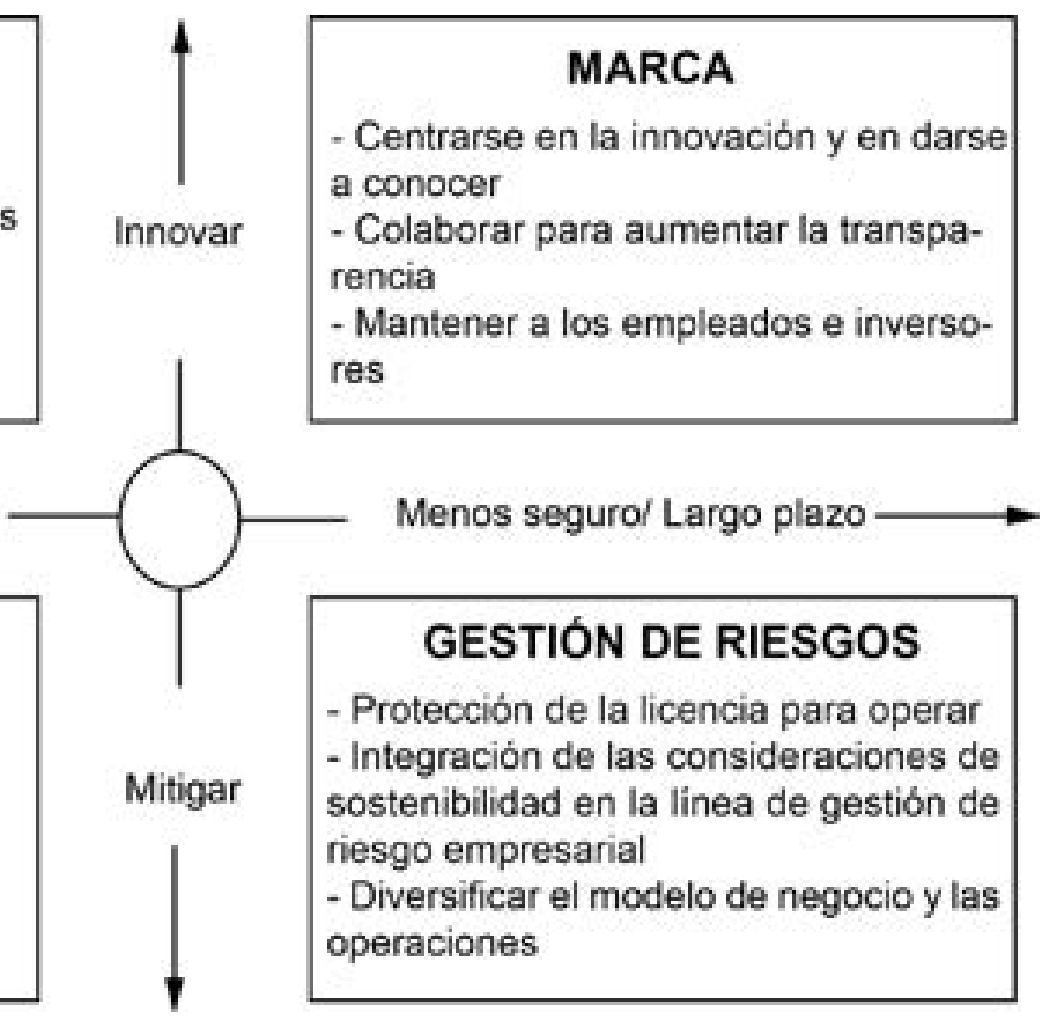

Figura 2. Cadena de suministro sostenible Fuente: Hanifan (2012) 
inventarios como para todo lo que se defina como un inventario se debe aplicar una adecuada gestión, la cual consiste en una serie de políticas y controles que monitorean los niveles de inventario y determinan los niveles que se deben mantener, el momento en que las existencias se deben reponer y el tamaño que deben tener los pedidos. Para ello, se implementa un sistema de inventarios, el cual, es responsable de ordenar y recibir los bienes; de coordinar la colocación de los pedidos y hacerle seguimiento al mismo. Además, el sistema debe mantener un control para responder a preguntas como: ¿El proveedor ha recibido el pedido?, ¿Éste ha sido despachado?, ¿Las fechas son correctas?, ¿Existen procedimientos para hacer un nuevo pedido o devolver la mercancía indeseable? (FIAEP, 20I4).

Costos de inventarios: La gestión de inventarios de acuerdo a FIAEP (20I4), implica dos costos básicos:

- Costos de penalización: por inexistencia de mercancías: son proporcionales a las ventas perdidas por inexistencia del producto, produce problemas de pérdida de imagen en la empresa.

- Costos de almacenamiento: representan los costos del capital inmovilizado como de gestión física y administrativa.

De acuerdo con Vidal (2009), los factores de costos que se evalúan en la gestión de inventarios son:

- Valor unitario del ítem: este valor se expresa en $\$$ / unidad, se representa con el símbolo v.

- Tasa de costo de llevar o mantener inventario: este costo comprende los costos de servicio del inventario (almacenamiento y manejo), el costo del espacio utilizado, los costos de capital y los costos de riesgo del inventario (los costos de obsolescencia, daños y filtraciones y, los seguros e impuestos). Es representado con el símbolo $r$.

- Costo de alistamiento: se encuentra simbolizado con una $A$ y corresponde a los costos de procesamiento, transmisión, manejo y compra de la orden de inventario. Cabe resaltar que se debe diferenciar entre un costo de ordenamiento para un comerciante y para un productor, ya que para el primero éste representa costos de preparación de formatos, correos, llamadas, autorización de pedidos, manejo de facturas y transporte de la orden; en tanto para un productor se refiere a los rubros relacionados con el montaje de maquinaria fija, transmisión y control de ordenes entre plantas, entre otros de alistamiento en el proceso productivo.
- $\quad$ Costo de faltante de inventario: se produce una vez se recibe una orden y no se cuenta con la cantidad de inventario suficiente para suplirla (Vidal, 2009).

\subsection{Selección de proveedores}

El principio fundamental de la selección de proveedores es la calidad, al considerar que la selección de proveedores es un proceso necesario en toda la organización o en la cadena de suministro. Generalmente, el departamento de compras es el encargado de la búsqueda y selección del proveedor que se ajusta en mayor medida a las necesidades de la empresa.

La selección de proveedores es considerada como un problema multicriterio, el cual incluye factores cuantitativos y cualitativos. Cabe resaltar que para seleccionar al mejor proveedor es necesario hacer una compensación entre estos factores tangibles e intangibles entre los que puede haber conflicto. Se considera que actualmente la selección de proveedores también se encuentra ligado a la optimización de costos y mejora de la variedad de la gama de productos para satisfacer las necesidades del consumidor.

En las técnicas tradicionales de selección de proveedores se cuenta como criterio de decisión el precio y se contrata con diferentes proveedores la compra de un mismo insumo o material, esto con la finalidad de evitar una dependencia del $100 \%$, con un solo proveedor, evitar el monopolio y obtener mejores condiciones al momento de llevar a cabo la negociación. Sin embargo, el enfoque actual de la gestión de proveedores procura mantener una relación estable con determinados proveedores. Se tiende a reducir el número de proveedores e incluso mantener relaciones con un proveedor único para un producto (Virseda, 20II).

En la Tabla I, Virseda (20II), presenta las ventajas del nuevo enfoque, tener uno $o$ varios proveedores, aunque el proceso de selección de proveedores puede llegar a ser muy distinto. Conforme se reduce el número de proveedores también aumenta la dependencia de ellos y el proceso de selección es más complejo y, por lo tanto, no es suficiente con desarrollar una lista estándar de criterios y aplicarla independientemente del proceso. Todo ello hace que aumente el coste asociada a la búsqueda de proveedor.

Para llevar a cabo una adecuada selección de proveedores se debe considerar:

- Evaluar las necesidades y definir los objetivos.

- Reunir un grupo de proveedores.

- Entrevista con los proveedores.

- Seleccionar y aplicar un método. 
Tabla I.

Comparación de alternativas en selección de proveedores.

\section{Varios Proveedores}

Se asegura la continuidad del suministro en caso de problemas.

Se evita el riesgo de excesiva dependencia del proveedor, si nos convertimos en su único cliente.

Se disminuyen los costes de cambio de proveedor

Posibilidad de contratar proveedores cuya capacidad no podría absorber toda la demanda.

\section{Proveedor Único}

Mayor facilidad para coordinar las relaciones y para manejar los flujos de materiales e información.

Menor tiempo y esfuerzo para promover relaciones más estrechas con el proveedor y para evaluar su actuación.

Calidad, plazos y servicios más uniformes.

Se mejora la responsabilidad del proveedor. Es posible utilizar mejor la capacidad de los proveedores,

Disminución de los costes de transporte y distribución, así como la posibilidad de reducir el inventario total en el proceso.

Posibilidad de concentrar útiles, herramientas e instalaciones específicas costosas en una sola fuente.

Fuente: Vírseda (20| I), p.2

Los criterios de evaluación de proveedores de acuerdo a Virseda (20II), se presentan en la Tabla 2, donde se hace una comparación que la mayoría de los autores que tratan este tema mencionan, el estudio de Dickson, el cual describe la importancia de 23 criterios clasificados con respecto a su importancia observada a comienzos de los sesenta.

En ese momento, los criterios más significativos eran calidad, entrega a tiempo, rendimiento y garantía y políticas de demanda. En el estudio de Virseda, se manejan 19 recursos y se consideraron 13 criterios. Todos ellos destacaron calidad como uno de los criterios que se debe tener en cuenta y más del $50 \%$ de los autores consideran coste, efectividad en la entrega y servicio como otros criterios importantes.

\subsection{Toma de decisiones bajo múltiples criterios}

La toma de decisiones bajo múltiples criterios se basa en que, para una adecuada toma de decisiones, se deben considerar diferentes metas, que permitan el alcance de objetivos globales de decisión. Al momento de llevar a cabo el ejercicio de toma de decisiones se debe identificar, si el problema es de solución estándar clásico, problemas difusos o problemas donde no existe un método de solución definido. El análisis multicriterio es una herramienta adecuada para este último grupo de problemas, debido a que permite considerar múltiples criterios de una manera explícita, estructurar el problema, así como ofrecer un modelo que ofrezca una decisión racional, explicada y justificada (Morales, 20I I).

Los elementos característicos de la toma de decisiones de
- Metas: es lo que se desea lograr en términos de estados específicos de tiempo y espacio.

- Objetivos: son los que indican la dirección en la que se debe enfocar el desarrollo del proyecto.

- Criterios: son los estándares de juicio o reglas que validan la aceptabilidad de la decisión.

- Atributos: son las características, cualidades o parámetros de desempeño de las alternativas a considerar para tomar la decisión.

- $\quad$ Alternativas: son las posibles decisiones que favoreceel logro de las metas establecidas.

\subsection{Clasificación de las técnicas multicritero}

Las técnicas multicriterio de acuerdo a Cabello (2017), se clasifican en dos grupos generales:

Las técnicas multicriterio discretas: son aquellas que utilizan una matriz decisional para el análisis de los diferentes criterios discretos. Para la elaboración de la matriz decisional deben definirse las diferentes alternativas, representadas como $\mathrm{m}$ y un conjunto de $\mathrm{n}$ alternativas; formando así una matriz $\mathrm{n} \times \mathrm{m}$. Cabe resaltar, que este enfoque guarda la consistencia en el ámbito teórico, siendo útil para contextos decisionales en los que el número de alternativas factibles sea reducido.

Entre las técnicas multicriterio discretas se tienen:

- Método de superación: método Electre (Elimination and choice translating algorithm): es un método que 
Tabla 2.

Criterios para la evaluación de proveedores.

\begin{tabular}{|c|c|c|}
\hline & Estudio de Dickson & Estudio de Virseda \\
\hline 1. & Calidad & 1. Calidad \\
\hline 2. & Entrega & 2. Precio/coste \\
\hline 3. & Rendimiento & 3. Efectividad en la entrega \\
\hline 4. & Garantía y políticas de demanda & 4. Servicio \\
\hline 5. & Capacidad de producción & 5. Situación financiera \\
\hline 6. & Precio & 6. Tiempo de entrega \\
\hline 7. & Capacidad Técnica & 7. Habilidad técnica \\
\hline 8. & Posición financiera & 8. Flexibilidad \\
\hline 9. & Cumplimiento de los procedimientos & 9. Capacidad de producción \\
\hline 10. & Sistema de comunicación & 10. Desarrollo \\
\hline 11. & Reputación y posición en la industria & 11. Actitud de gestión \\
\hline 12. & Deseo de negocio & 12. Tasa de cumplimiento \\
\hline 13. & Administración y organización & 13. Localización geográfica \\
\hline 14. & Control de funcionamiento & \\
\hline 15. & Servido de reputación & \\
\hline & Actitud & \\
\hline & Impresión & \\
\hline 18. & Habilidad de embalaje & \\
\hline 19. & Relaciones laborales & \\
\hline 20. & Localización geográfica & \\
\hline & Cantidad de negocios anteriores & \\
\hline 22. & Formación & \\
\hline 23. & Acuerdos recíprocos & \\
\hline
\end{tabular}

Fuente: Vírseda (20I I), p.2.

ha sido reformado con la finalidad de obtener mejores resultados; existen entonces el Electre II, Electre III y Electre IV. Estos métodos se basan en el axioma de comparabilidad parcial fundamental. Cada estructura de preferencia utilizada recoge diferentes tipos de criterios de acuerdo a la complejidad de formulación y la naturaleza del problema.

El método Electre es una técnica que permite reducir el tamaño, el conjunto de soluciones eficientes, mediante la participación de subconjuntos. Adicionalmente, esta técnica utiliza pseudocriterios, matriz decisional, matriz de concordancia, matriz de discordancia,y grafo de superación del método

- Método de jerarquía analítica (AHP-Analytic Hierarcgy Process): Es un método que combina aspectos tangibles e intangibles con el fin de obtener en una escala de razón, las prioridades asociadas a las diferentes alternativas.
Según Saaty (2008), el proceso de jerarquía analítica (AHP), es una teoría de medición a través de comparaciones por pares y se basa en los juicios de expertos para derivar escalas de prioridad. Son estas escalas las que miden los intangibles en términos relativos. Las comparaciones se realizan utilizando una escala de juicios absolutos que representa, cuánto más, un elemento dominio, a otro con respecto a un determinado atributo. Los juicios pueden ser inconsistentes y cómo medir inconsistencias y mejorar los juicios, esa es una preocupación del AHP, por ello, hay que hacer cuanto sea posible para mejorar la consistencia. Las escalas de prioridad derivadas son sintetizadas, multiplicando por la prioridad de sus nodos principales.

En otras palabras, esta técnica se caracteriza por modelar el problema de forma gráfica, para ello, utiliza una estructura jerárquica. Adicionalmente, usa una comparación por pares y una escala válida para la toma de decisiones. El AHP se caracteriza por facilitar la organización de información de los problemas, su descomposición, análisis por partes y sintonización. 
Esta herramienta consta de cuatro axiomas: el primero consiste en la intensidad de preferencias; el segundo indica que los elementos que son comparados deben ser del mismo orden de magnitud respecto a una misma propiedad; el tercero dice, que es posible determinar y controlar el tipo de dependencia de los elementos de dos niveles consecutivos en la jerarquía y dentro del mismo nivel, el cuarto axioma consiste en que las expectativas deben estar representadas en la estructura en términos de criterios y alternativas. Al considerar estos axiomas se determina la formulación y resolución del problema de decisión como una jerarquía y se hacen explícitos los juicios en forma de comparación por pares.

- Método axiomático de Arroe - Raynaud: Este método asocia los problemas multicriterio con la teoría de la elección social, donde se considera que cada criterio es un agente social, generando así una correlación perfecta entre los problemas y la elección social.

Las técnicas multicriterio continuas: se caracterizan porque la cantidad de alternativas que considera el decisor son infinitas. Esta clase de técnicas son utilizadas en ambientes de certeza, es decir, que se cuenta con información completa acerca de los estados de la naturaleza que se van a presentar en momentos posteriores. Cabe resaltar que, en ocasiones, no se hace posible optimizar simultáneamente todos los objetivos, debido a que se presentan conflictos entre los mismos o se carece de recursos necesarios para la ejecución.

- Matriz de pagos: Es un método que permite cuantificar el nivel de conflicto existente entre los objetivos considerados. La dimensión de la matriz coincide con el número de objetivos y se obtiene mediante la optimización individual de éstos, calculando los valores obtenidos por el resto de objetivos en cada solución óptima. En la diagonal principal de la matriz se identifican dos puntos principales uno es el punto ideal $y$ el otro el punto anti-ideal.

Técnicas generadoras del conjunto eficiente: Es una técnica que se caracteriza por extraer las soluciones eficientes del conjunto de soluciones factibles. Estas soluciones eficientes son denominadas óptimas de Pareto y son aquellas en las que no se puede alcanzar una mejora en el objetivo sin que empeore ningún otro. Entre estas técnicas se encuentra el método de las ponderaciones, el método de las e restricciones y el método simplex multicriterio.

Técnicas con información a priori: Se caracterizan 32 preferencias del decisor. El objetivo de estas técnicas es determinar las soluciones que mejor se adapten a dichas preferencias.

\subsubsection{Aplicaciones de la toma de decisiones bajo múltiples criterios}

Debido a que se considera que la toma de decisiones es fundamental en la búsqueda de la eficiencia y productividad de las empresas, de los sectores industriales y de las regiones; se utilizan las técnicas multicriterio para la toma de decisiones en dichos escenarios, esto por sus características entre las que se encuentra la intervención de múltiples variables o criterios de selección.

Según Cabello (2017), algunas de las aplicaciones de la toma de decisiones bajo múltiples criterios son: selección de carteras, licitación pública, determinación del impacto ambiental y valoración de los activos medioambientales, reemplazo de los equipos industriales, la selección de proveedores, entre otras.

- Selección de carteras: en este caso los métodos multicriterio permiten identificar la cartera de activos óptima para el conjunto de criterios tenidos en consideración. Entre las técnicas aplicadas a este caso específico se encuentran las desarrolladas por Baumol, Brewster y Philippatos, así como la programación por metas y el proceso analítico jerárquico (AHP).

- Licitación pública: en esta aplicación se estudia cual es el mejor proyecto, además, que ahorre recursos al estado. Los métodos más utilizados consisten en establecer una serie de criterios y su peso relativo; también se utiliza el método AHP mediante la aplicación de números fuzzy.

- Determinación del impacto ambiental y valoración de los activos medioambientales: los métodos multicriterio son útiles para la determinación de alternativas, identificación y valoración de los impactos ambientales; permitiendo obtener las mejores soluciones para el conjunto de objetivos. Una de las metodologías utilizadas para el desarrollo de estas soluciones es el método AHP.

- Reemplazo de equipos industriales: este estudio surgió debido a la necesidad de determinar el momento óptimo para el reemplazo de los equipos industriales, este problema nace en el año 1933 y desde entonces se ha realizado enriquecedores estudios. El contexto de esta situación indica que los modelos de reemplazo tienen una naturaleza de múltiples metas y objetivos por alcanzar, motivo por el cual las técnicas multicrite- 
rio son de alta utilidad, permitiendo la incorporación de variables tecnológicas y técnicas al análisis. Entre las técnicas utilizadas se encuentran la de indicador integral de reemplazo (IIR) y el método AHP.

- Selección de proveedores: esta es una de las actividades significativas para el correcto funcionamiento de las compañías. Para la selección del proveedor se deben considerar aspectos diferentes al precio, incluyendo así factores de calidad, fiabilidad y confianza. De acuerdo a esto, se indica entonces, que la decisión de selección de proveedor corresponde al ámbito multicriterio y de impacto estratégico. Se considera que una de las metodologías multicriterio más acertada para este caso es el método AHP, el cual ha sido implementado por diferentes autores. Otras técnicas utilizadas son el método Electre II y la programación por objetivos difusa.

\section{Metodología}

Son diversos los métodos y/o mecanismos existentes en la literatura y en la práctica, para la selección de proveedores. Por lo tanto, es necesario que cada empresa evalúe cual es el mecanismo que mejor se ajusta a su operación y capacidad para hacer la toma de decisiones más apropiada a sus necesidades.

Para efectos prácticos y atendiendo al conocimiento y habilidad del recurso humano de la empresa caso de estudio, se decidió que el método más apropiado para este caso era el proceso analítico jerárquico (AHP). Esto debido al conocimiento y la facilidad en la transferencia del mismo, por parte de los autores; $y$, al manejo y practicidad para los expertos que intervinieron en el problema.

Además, el AHP se caracteriza por facilitar la organización de información de los problemas, su descomposición, análisis por partes y sintonización; haciendo mucho más fácil el manejo y aplicación del método.

Adicionalmente, para aplicar el método, se consideró lo siguiente:

A partir de un listado de insumos críticos, de la empresa caso de estudio del año 2014 y de acuerdo a criterios del área de calidad, que tenían que ver con insumos indispensables en cantidad y calidad, se escogieron los tres insumos más críticos.

Para ello, se pidió a un grupo de expertos del área de calidad, hacer una selección de los insumos más importantes de acuerdo a las condiciones y políticas de sostenibilidad de la compañía considerando aspectos como productividad, inocuidad y calidad.
De acuerdo a esas condiciones en términos de sostenibilidad, se determinó que el insumo más crítico era el hidróxido de calcio, seguido de una serie de compuestos químicos entre los cuales se fundamentó su selección, en los niveles de consumo y contaminación que podían producir, además de la importancia dentro del sistema productivo de la empresa.

Para dar alcance a este trabajo y desarrollar el modelo de selección, se escogieron los tres insumos más críticos en el proceso productivo. Determinando, por tanto, que los insumos a valorar serían:

- $\quad$ Hidróxido de calcio $\mathrm{Ca}(\mathrm{OH}) 2$

- Profloc 932 (floculante)

- Alcohol anhidro

Alternativas de proveedores: Después de haber seleccionado los tres insumos para el desarrollo del trabajo, se procedió a analizar los posibles proveedores de dichos insumos teniendo en cuenta que cumplieran con las condiciones mínimas exigidas por la empresa. Entre las condiciones analizadas se tuvieron: información general del proveedor, ubicación, precio contra competencia, legalidad, derechos humanos, medio ambiente, prácticas laborales, prácticas éticas. En la Tabla 3, se aprecian los proveedores para los insumos.

Tabla 3.

Proveedores por insumo.

\begin{tabular}{ccc}
\hline Criticidad & Insumo & Proveedor \\
\hline \multirow{2}{*}{1} & Hidróxido de calcio & PRODECALES S.A. \\
& $\mathrm{Ca}(\mathrm{OH})_{2}$ & CALTEK S.A.S \\
& & PROTECNICA \\
& & INGENIERIA SAS \\
& & CHEMICAL \\
2 & Profloc 932 (floculante) & COACHING \\
& & SERVICES SAS \\
& & LIPESA COLOMBIA \\
& & SA \\
& & CONQUIMICA \\
& & SUCROAL S.A. \\
\hline
\end{tabular}

Fuente: Elaboración propia

Luego de haber definido los insumos de trabajo, los posibles proveedores y el método AHP, se procedió a hacer la aplicación de dicho método con los datos del estudio. Para ello, se desarrollaron los siguientes pasos, que se toman como una adaptación de lo propuesto por García y Lamata (2009).

Paso I: Definición del objetivo general. Consiste en indicar el objetivo general del problema e identificar los criterios y/o subcriterios que más influyen. 
Paso 2: Definición del árbol de decisión: Consiste en crear el árbol de decisión con criterios, subcriterios y alternativas del sistema de selección.

Paso 3: Definición expertos que evalúan el sistema: Consiste en la definición de los expertos que con sus juicios valoran el sistema comparando importancias entre criterios, subcriterios y alternativas.

Paso 4: Evaluación por parte de los expertos: Consiste en la valoración de los expertos a las comparaciones por pares entre criterios, subcriterios y alternativas de acuerdo al árbol de decisión de cada insumo.

Paso 5: Selección de proveedores: Consiste en seleccionar el mejor proveedor para cada insumo a partir de la ponderación entre el peso global de los subcriterios y el peso de cada alternativa en los mismos.

\section{Resultados}

De acuerdo a Sarache, Castrillón y Ortiz (2009), la selección de proveedores es una decisión de múltiples criterios y de impacto estratégico en las organizaciones. Igualmente, muestran que el paradigma del múltiple criterio se presenta como una ayuda efectiva en la práctica de la toma de decisiones y gestión de las organizaciones; por tanto, la selección de una herramienta multicriterio es de gran ayuda en la selección de proveedores para las empresas.

En la actualidad, la empresa caso de estudio, toma las decisiones de selección de proveedores basada en métodos

empíricos, por tanto, los resultados que se presentan de esta investigación son pertinentes, puesto que pretenden guiar a la empresa hacia una mejora significativa en los procesos de gestión y calidad, que le permitirán ser más competitiva en su sector.

Para efectos prácticos se desarrollaron los pasos mencionados, de la siguiente manera:
Paso I: Definición del objetivo general. En tal sentido, el objetivo general en este caso fue seleccionar el proveedor más adecuado para cada insumo correspondiente y, los criterios y subcriterios. De acuerdo a lo establecido por la empresa caso de estudio.

Para la selección de criterios y subcriterios, se tuvo en cuenta lo establecido por la empresa, en sus políticas de calidad y sostenibilidad, que buscan mejorar su competitividad. En los cuales las variables de sostenibilidad estaban asociadas con la viabilidad económica, medio ambiente y cumplimiento legal. Igualmente, en los criterios de calidad, se tomaron en cuenta el cumplimiento en general de las especificaciones, los plazos de entrega y el precio. Además, de criterios de mercado, como capacidad de adaptación e innovación. En la Tabla 4, se muestran estos criterios y subcriterios:

Paso 2: Definición del árbol de decisión. En este paso, se llevó a cabo la valoración de los elementos a través de la comparación por pares. En esta etapa se utilizó la escala de valoración propuesta por Saaty (citado en García-Gomez. 2013) (Ver Tabla 5).

En la Figura 3, se observa la relación del árbol de decisión del insumo hidróxido de calcio con los criterios, subcriterios y posibles proveedores; en la Figura 4 , se observa el árbol de decisión del insumo alcohol anhidro, con los criterios, subcriterios y posibles proveedores, y en la Figura 5 , se observa el árbol de decisión del insumo floculante, con los criterios, subcriterios y posibles proveedores.

Paso 3: Definición expertos que evalúan el sistema. Los expertos se seleccionaron, teniendo en cuenta, que eran los involucrados directos, en la toma de decisión de la compra de insumos.

- El: Jefe de compras

- E2: Jefe de Calidad

- E3: Jefe de producción

Tabla 4.

Criterios y subcriterios del sistema.

\section{CRITERIOS}

\begin{tabular}{cccc}
\hline & De Sostenibilidad & De Mercado & De Calidad \\
\hline VUBCRITERIOS & Cumplimiento de ley & Capacidad de adaptación & Cumplimiento especificaciones \\
& Gestión ambiental & Innovación & Plazo de entrega \\
& & & Precio \\
\hline
\end{tabular}


Tabla 5.

Escala de valoración de Saaty

\begin{tabular}{cll}
\hline Escala Numérica & Escala Verbal & Explicación \\
\hline 1 & Igual importancia & El criterio A es igual de importante que el criterio B \\
3 & Importancia moderada & La experiencia y el juicio favorecen ligeramente al criterio A sobre el B \\
7 & Importancia grande & La experencia y el juicio favorecen fuertemente el criterio A sobre el B \\
9 & Importancia muy grande & El criterio A es mucho más importante que el B \\
$2,4,6$, y 9 & Importancia extrema & La mayor importancia del criterio A sobre el B esta fuera de toda duda \\
\hline
\end{tabular}

Fuente: García (2013).

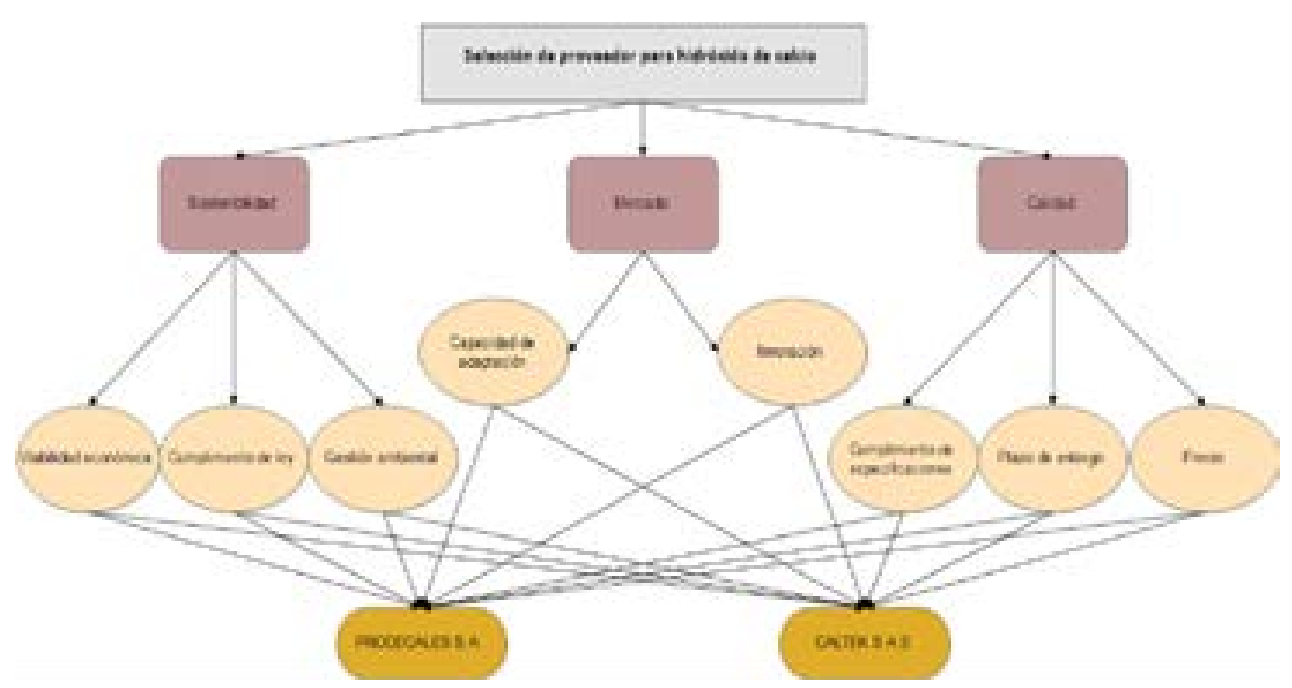

Figura 3. Árbol de decisión hidróxido de calcio

Fuente: Elaboración propia

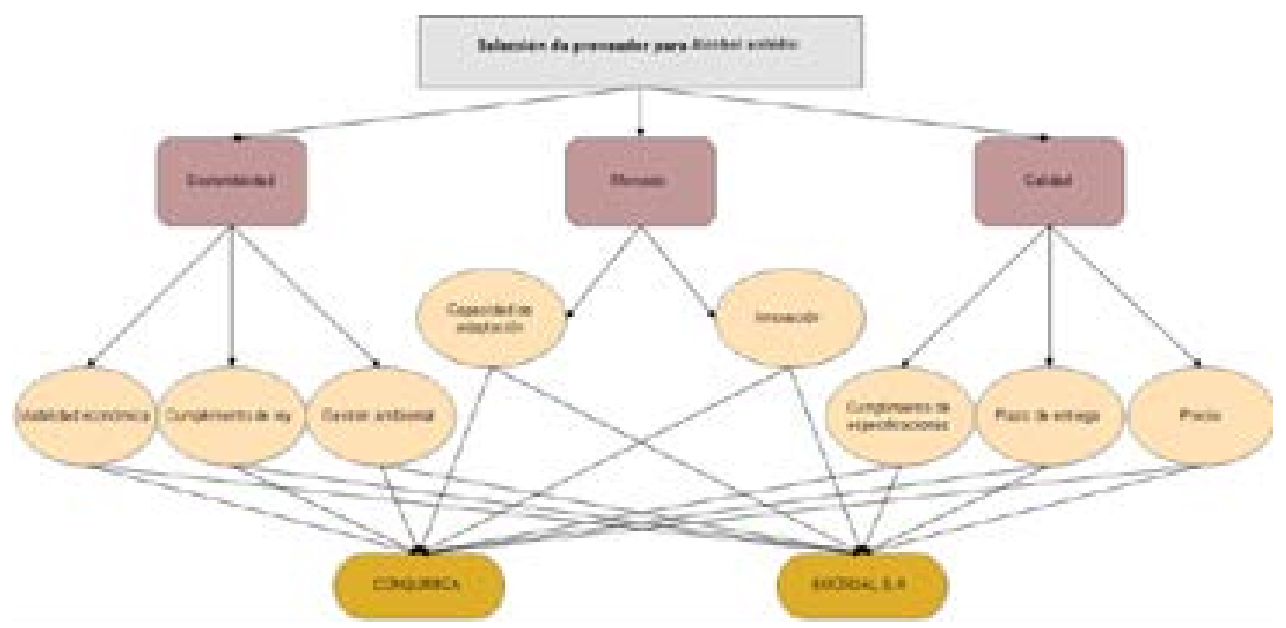

Figura 4. Árbol de decisión alcohol anhidro

Fuente: Elaboración propia 


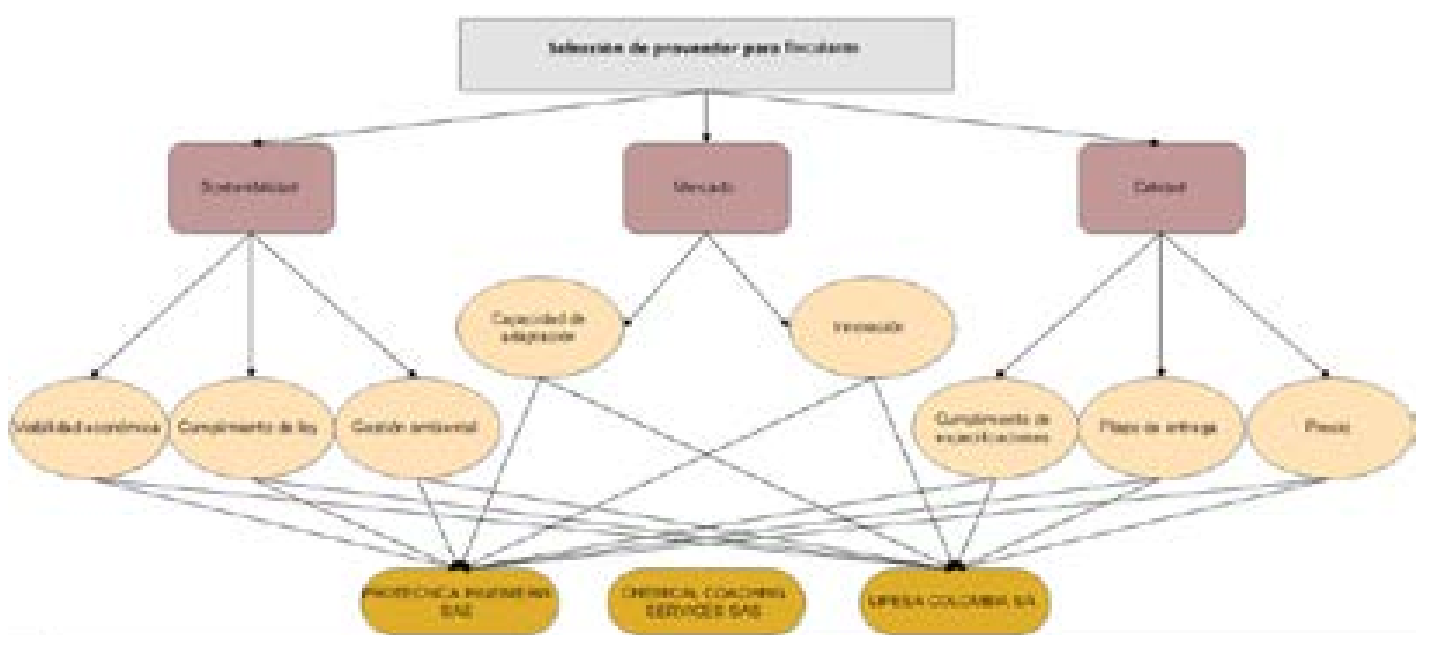

Figura 5. Árbol de decisión floculante Fuente: Elaboración propia

Paso 4: Evaluación por parte de los expertos. En este caso, se presentó a cada uno de los expertos, un conjunto de matrices en las que inicialmente compararon los criterios entre ellos mismos, posteriormente los subcriterios de cada criterio y finalmente las alternativas para cada subcriterio.

Para efectos de guía en la valoración de los expertos, en la Tabla 6 , se presentan las notaciones que se usaron en las matrices para referirse a los criterios y subcriterios; en la Tabla 7, se presentan las notaciones que se usaron en las matrices para referirse a las alternativas del insumo hidróxido de calcio; en la Tabla 8 , se presentan las notaciones que se usaron en las matrices para referirse a las alternativas del insumo floculante, y en la Tabla 9 , se presentan las notaciones que se usaron en las matrices para referirse a las alternativas del insumo alcohol anhidro.

\section{Tabla 6.}

Notación para criterios y subcriterios.

\begin{tabular}{|c|c|c|}
\hline \multicolumn{2}{|r|}{ SUBCRITERIOS } & \multirow{2}{*}{ Criterios } \\
\hline Notación & Descripción & \\
\hline $\mathrm{SC} 1.1$ & Viabilidad Económica & \multirow{3}{*}{ Sostenibilidad C1 } \\
\hline $\mathrm{SC} 1.2$ & Cumplimiento de ley & \\
\hline $\mathrm{SC} 1.3$ & Gestión ambiental & \\
\hline $\mathrm{SC} 2.1$ & Capacidad de adaptación & \multirow{3}{*}{ Mercado C2 } \\
\hline $\mathrm{SC} 2.2$ & Innovación & \\
\hline SC3.1 & Cumplimiento especificaciones & \\
\hline $\mathrm{sc} 3.2$ & Plazo de entrega & \multirow[t]{2}{*}{ Calidad C3 } \\
\hline Sc3.3 & Precio & \\
\hline
\end{tabular}

Fuente: Elaboración propia

Tabla 7.

Notación para alternativas del insumo I (hidróxido de calcio).

\begin{tabular}{cc}
\hline Notación & Descripción \\
\hline Al1.1 & PRODECALES S.A. \\
Al1.2 & CALTEK S.A.S \\
\hline
\end{tabular}


Tabla 8.

Notación para alternativas del insumo 2 (Floculante).

\begin{tabular}{cc}
\hline Notación & Descripción \\
\hline Al2.1 & PROTECNICA INGENIERIA SAS \\
Al2.2 & CHEMICAL COACHING SERVICES SAS \\
Al2.3 & LIPESA COLOMBIA SA \\
\hline
\end{tabular}

Fuente: Elaboración propia

Tabla 9.

Notación para alternativas del insumo 3 (Alcohol anhidro).

\begin{tabular}{cc}
\hline Notación & Descripción \\
\hline Al3.1 & CONQUIMICA \\
Al3.2 & SUCROAL S.A. \\
\hline
\end{tabular}

Fuente: Elaboración propia

Después de haber socializado las notaciones de guía a cada uno de los expertos, se procedió a entrevistarlos de manera individual y se les pidió que, de acuerdo a su experiencia, realizaran la comparación por pares. Esto se fue registrando en cada una de las plantillas del sistema definido. El proceso se realizó en un intervalo de un mes, con sesiones de 2 horas semanales con cada uno de los expertos.

Cabe anotar en este punto que, para la valoración, tanto de criterios, como de subcriterios y alternativas, se realizó un diseño propio de las plantillas en MS EXCEL.

También es importante aclarar, que en la valoración de los criterios y los subcriterios se determina la importancia de estos frente al objetivo-meta, mientras que en las alternativas es la preferencia que da el decisor de cada una de éstas con respecto a los criterios.

Valoración de criterios: Como primera parte de la evaluación, se solicitó a los tres expertos valorar la matriz de comparación de criterios.

En la Tabla 10 , se evidencia la valoración comparativa para los pares de criterios realizada por el experto I. En la Tabla $\underline{I}$, se presenta la valoración comparativa para los pares de criterios realizada por el experto 2 y en la Tabla 12 , la valoración comparativa para los pares de criterios realizada por el experto 3.

Tabla 10.

Comparación de criterios - Experto I.

\begin{tabular}{cccc}
\hline & Sostenibilidad & Mercado & Calidad \\
\hline Sostenibilidad & 1 & 0,11111111 & 0,14285714 \\
Mercado & 9 & 1 & 3 \\
Calidad & 7 & 0,33333333 & 1 \\
TOTAL & 17 & 1,44444444 & 4,14285714 \\
\hline
\end{tabular}

Fuente: Elaboración propia

Tabla II.

Comparación de criterios - Experto 2.

\begin{tabular}{cccc}
\hline & Sostenibilidad & Mercado & Calidad \\
\hline Sostenibilidad & 1 & 3 & 0,14285714 \\
Mercado & 0,333333333 & 1 & 0,111111111 \\
Calidad & 7 & 9 & 1 \\
TOTAL & 8,333333333 & 13 & 1,25396825 \\
\hline
\end{tabular}

Fuente: Elaboración propia 
Comparación de criterios - Experto 3.

\begin{tabular}{cccc}
\hline & Sostenibilidad & Mercado & Calidad \\
\hline Sostenibilidad & 1 & 0,33333333 & 0,33333333 \\
Mercado & 3 & 1 & 1 \\
Calidad & 3 & 1 & 1 \\
TOTAL & 7 & 2,33333333 & 2,33333333 \\
\hline
\end{tabular}

Fuente: Elaboración propia

Posteriormente, se consolidaron las valoraciones en una sola matriz de pares de criterios. Para ello, se hizo la productoria de las 3 matrices, obteniendo como resultado la matriz de la Tabla 13.

En cuanto a la matriz consolidada, a cada valor de la matriz se le elevó a una potencia de $\mathrm{I} / \mathrm{n}$, donde $\mathrm{n}$ es el número de expertos, en este caso 3. Así pues, la matriz consolidada de criterios quedó como se muestra en la Tabla 14.

Después de obtener la matriz consolidada se procedió a normalizar dicha matriz, de tal manera, que cada valor se divide por el total de su columna correspondiente. Así, se obtuvo la Tabla 15.

Tabla 13.

Productoria de matices de expertos.

\begin{tabular}{cccc}
\hline PRODUCTORIA & Sostenibilidad & Mercado & Calidad \\
\hline Sostenibilidad & 1 & 0,111111111 & 0,00680272 \\
Mercado & 9 & 1 & 0,33333333 \\
Calidad & 147 & 3 & 1 \\
TOTAL & 157 & 4,111111111 & 1,34013605 \\
\hline
\end{tabular}

Fuente: Elaboración propia

Tabla 14.

Matriz consolidada de criterios.

\begin{tabular}{cccc}
\hline CONSOLIDADA & Sostenibilidad & Mercado & Calidad \\
\hline Sostenibilidad & 1 & 0,48074986 & 0,18947891 \\
Mercado & 2,080083823 & 1 & 0,69336127 \\
Calidad & 5,277632088 & 1,44224957 & 1 \\
TOTAL & 8,357715911 & 2,92299943 & 1,88284019 \\
\hline
\end{tabular}

Fuente: Elaboración propia

Tabla 15.

Matriz normalizada de criterios.

\begin{tabular}{cccc}
\hline Normalización & Sostenibilidad & Mercado & Calidad \\
\hline Sostenibilidad & 0,11964992 & 0,16447142 & 0,10063462 \\
Mercado & 0,24888185 & 0,34211433 & 0,24873096 \\
Calidad & 0,63146823 & 0,49341425 & 0,74619289 \\
\hline
\end{tabular}


Para finalizar, al hacer el promedio de cada fila de la matriz normalizada, se obtuvo el vector de pesos para cada uno de los criterios (Ver Tabla 16). De acuerdo con la información de la Tabla, se puede decir que el peso de los criterios del sistema es de $62,3 \%$ para calidad, $27,9 \%$ para mercado y $12,8 \%$ para sostenibilidad.

Valoración de subcriterios: Para realizar la valoración de subcriterios se procedió de la misma manera que con los criterios, teniendo en cuenta que se debe comparar entre pares de subcriterios, que correspondan al mismo criterio. Así pues, los vectores de pesos de subcriterios para cada uno de los criterios se muestran en Tablas 17, 18 y 19.

En la Tabla 17, se presentan los vectores de peso del subcriterio de sostenibilidad, se puede decir que el peso de los subcriterios del criterio sostenibilidad es de $47,2 \%$ para gestión ambiental, $27,5 \%$ para viabilidad económica y $25,1 \%$ para cumplimiento de ley.

En la Tabla 18, se presentan los vectores de peso del subcriterio de mercado, de acuerdo con la información, se evidencia que el peso de los subcriterios del criterio mercado es de $67,5 \%$ para innovación y $32,4 \%$ para capacidad de adaptación.

En la Tabla 19, se presentan los vectores de peso del subcriterio de calidad. De acuerdo con esta información se puede decir que el peso de los subcriterios del criterio calidad es de $41,8 \%$ para plazo de entrega; $30,7 \%$ para precio y $27,4 \%$ para cumplimiento especificaciones.

Tabla 16.

Vector de pesos de criterios.

\begin{tabular}{lcc} 
& CRITERIOS & Vector de Pesos \\
\hline & Sostenibilidad & 0,12825199 \\
& Mercado & 0,27990905 \\
& Calidad & 0,62369179 \\
\hline Fuente: Elaboración propia & & \\
Tabla 17. & Sostenibilidad & Vector de Peso \\
\hline & Sc1.1 & 0,27593091 \\
\hline SC1.2 & 0,25115146 \\
\hline & Sc1.3 & 0,47291763 \\
\hline
\end{tabular}

Fuente: Elaboración propia

Tabla 18.

Vector de pesos de subcriterios de mercado.

\begin{tabular}{ccc}
\hline Mercado & Vector de Peso \\
\hline SC2.1 & 0,32466649 \\
SC2.2 & 0,67533351 \\
\hline
\end{tabular}

Fuente: Elaboración propia

Tabla 19.

Vector de pesos de subcriterios de calidad.

\begin{tabular}{cl}
\hline Calidad & Vector de Peso \\
\hline SC3.1 & 0,27428337 \\
SC3.2 & 0,41849348 \\
SC3.3 & 0,30722315 \\
\hline
\end{tabular}


Dado que el último nivel de los árboles de decisión en el que se puede comparar las alternativas, es el de los subcriterios, se procedió a realizar el cálculo del peso global (w), dentro el sistema de cada uno de los subcriterios. Lo anterior se logró, multiplicando los pesos de los subcriterios dentro de cada criterio, por el respectivo peso del criterio dentro del sistema (VerTabla 20).

De acuerdo a la Tabla 20, el subcriterio con mayor peso en el sistema es el plazo de entrega con un $26,1 \%$; seguido del precio con un $19,1 \%$; por su parte el subcriterio de menor peso en el sistema es el cumplimiento de ley con un $3,2 \%$.

Es importante aclarar que esta valoración de criterios y subcriterios es la misma para los tres insumos seleccionados.

Valoración de alternativas: Para realizar la valoración de alternativas se procedió de la misma manera que con los criterios y subcriterios, teniendo en cuenta que se debía comparar entre pares de alternativas, para cada uno de los subcriterios de último nivel.
Así pues, los vectores de pesos de las alternativas para cada uno de los subcriterios se muestran en las Tablas 21 , 22 y 23 correspondientes a cada uno de los 3 insumos seleccionados (Ver Tablas 21, 22 y 23).

De acuerdo a la Tabla 21, la alternativa Caltek S.A.S es mejor en 5 subcriterios y la alternativa Prodecales S.A, es mejor en 3 subcriterios.

En la Tabla 22, se presentan los vectores de pesos de las alternativas del $\mathrm{f}$ loculante para cada uno de los subcriterios y de acuerdo con los datos presentados en dicha tabla, la alternativa Chemical coaching services SAS es mejor en 5 subcriterios y la alternativa Lipesa Colombia S.A. es mejor en 3 subcriterios.

En la Tabla 23, se presentan los vectores de pesos de las alternativas del alcohol anhidro, para cada uno de los subcriterios y con base en los datos presentados en dicha tabla, la alternativa Conquímica es mejor en 4 subcriterios y la alternativa Sucroal S.A. es mejor en 3 subcriterios.

Tabla 20.

Pesos globales de los subcriterios.

\begin{tabular}{|c|c|c|c|c|}
\hline & Sostenibilidad & Mercado & Calidad & \multirow{2}{*}{$\mathbf{W}$} \\
\hline & 0,128251985 & 0,27990905 & 0,62369179 & \\
\hline SC1.1 & 0,275930914 & & & 0,03538869 \\
\hline Sc1.2 & 0,251151459 & & & 0,03221067 \\
\hline $\mathrm{sc} 1.3$ & 0,472917628 & & & 0,06065262 \\
\hline SC2.1 & & 0,32466649 & & 0,09087709 \\
\hline $\mathrm{sc} 2.2$ & & 0,67533351 & & 0,18903196 \\
\hline sc3.1 & & & 0,27428337 & 0,17106829 \\
\hline $\mathrm{sc} 3.2$ & & & 0,41849348 & 0,26101095 \\
\hline $\mathrm{sc} 3.3$ & & & 0,30722315 & 0,19161255 \\
\hline
\end{tabular}

Fuente: Elaboración propia

Tabla 21.

Pesos de las alternativas del hidróxido de calcio para cada subcriterio.

\begin{tabular}{ccccccccc}
\hline Insumo 1 & SC1.1 & SC1.2 & SC1.3 & SC2.1 & SC2.2 & SC3.1 & SC3.2 & SC3.3 \\
\hline Al1.1 & 0,2194 & 0,2194 & 0,2500 & 0,7806 & 0,8251 & 0,1593 & 0,4512 & 0,8483 \\
Al1.2 & 0,7806 & 0,7806 & 0,7500 & 0,2194 & 0,1749 & 0,8407 & 0,5488 & 0,1517 \\
\hline
\end{tabular}


Tabla 22.

Pesos de las alternativas del floculante para cada subcriterio.

\begin{tabular}{ccccccccc}
\hline Insumo 2 & SC1.1 & SC1.2 & SC1.3 & SC2.1 & SC2.2 & SC3.1 & SC3.2 & SC3.3 \\
\hline Al2.1 & 0,0997 & 0,0935 & 0,0906 & 0,1133 & 0,1424 & 0,0826 & 0,0770 & 0,1073 \\
Al2.2 & 0,5216 & 0,5727 & 0,5823 & 0,5094 & 0,3512 & 0,5603 & 0,3236 & 0,1904 \\
Al2.3 & 0,3787 & 0,3338 & 0,3271 & 0,3772 & 0,5065 & 0,3571 & 0,5994 & 0,7024
\end{tabular}

Fuente: Elaboración propia. (Al 2.I: Protecnica; Al2.2: Chemical Coaching; Al2.3: Lipesa).

Tabla 23.

Pesos de las alternativas del alcohol anhidro para cada subcriterio.

\begin{tabular}{ccccccccc}
\hline Insumo 3 & SC1.1 & SC1.2 & SC1.3 & SC2.1 & SC2.2 & sC3.1 & SC3.2 & SC3.3 \\
\hline Al3.1 & 0,7806 & 0,8083 & 0,7115 & 0,2194 & 0,2500 & 0,7115 & 0,5000 & 0,1917 \\
Al3.2 & 0,2194 & 0,1917 & 0,2885 & 0,7806 & 0,7500 & 0,2885 & 0,5000 & 0,8083 \\
\hline
\end{tabular}

Fuente: Elaboración propia. (AI3. I: Conquímica; Al3.2: Sucroal).

Paso 5: Selección de proveedores: En las siguientes Tablas se observan los resultados finales del método de selección para los respectivos insumos.

En la Tabla 24, se presenta el resultado final, para seleccionar el proveedor del hidróxido de calcio y de acuerdo a lo presentado en esta, el proveedor seleccionado para el hidróxido de calcio, es Prodecales S.A, con un peso del 56\%.

En la Tabla 25, se presenta el resultado final, para seleccionar el proveedor del floculante. De acuerdo con la información de la Tabla, el proveedor seleccionado para el floculante, es Chemical coaching services SAS con un peso del 53\% (Ver Tabla 25).

En la Tabla 26, se presenta el resultado final, para seleccionar el proveedor del alcohol anhidro el cual fue Sucroal SA con un peso del 58\% (Ver Tabla 26).

De acuerdo a la aplicación de la metodología, se puede concluir que la empresa caso de estudio, debe seleccionar para el insumo hidróxido de calcio el proveedor Prodecales, para el insumo Floculante Profloc 932 el proveedor Chemical Coaching Services SAS y para el insumo Alcohol Anhidro el proveedor Sucroal S.A.

Tabla 24.

Resultado final para el hidróxido de calcio.

\begin{tabular}{cc}
\hline ALTERNATIVA & PESO \\
\hline Al1.1 & $56 \%$ \\
Al1.2 & $47 \%$ \\
\hline
\end{tabular}

Fuente: Elaboración propia. (All.I: Prodecales. All.2: Caltek)

Tabla 25.

Resultado final para el floculante.

ALTERNATIVA

$\mathrm{AI} 2.1$

Al2.2

Al2.3
PESO

$10 \%$

$40 \%$

$53 \%$

Fuente: Elaboración propia. (Al 2.I: Protecnica;Al2.2: Chemical Coaching; Al2.3: Lipesa). 
Tabla 26.

Resultado final para el alcohol anhidro.

ALTERNATIVA

Al3.1

Al3.2
PESO

$45 \%$

$58 \%$

Fuente: Elaboración propia. (Al3. I: Conquímica; Al3.2: Sucroal).

\section{Discusión de resultados}

La metodología multicriterio es una herramienta que permite de una manera ordena y gráfica, la solución a problemas que tienen o requieren el manejo de diversas variables, para su solución. Además, con la información suministrada se pueda hacer un análisis de la importancia de aspectos relevantes y la interacción entre los mismo, a través de la realización de un modelo jerárquico formado por tres niveles que son, las metas, los criterios y las alternativas.

Estos análisis igualmente, requieren no sólo del conocimiento de la misma metodología, sino del concurso de expertos en el tema o problema a tratar, con quienes se debe trabajar de manera mancomunada, para la solución del mismo.

Después de determinar cuál era el problema a solucionar y que esta metodología era la mejor ayuda para dar solución al problema, se procedió a determinar unos criterios, subcriterios y alternativas, que ayudaron a los expertos de acuerdo a su conocimiento sobre el tema, a aportar una serie de soluciones que trajo consigo, un peso o porcentaje, de su conocimiento sobre el tema que, de otra manera, no se podría determinar solo con el uso de operaciones matemáticas.

La metodología aplicada permitió identificar diversas relaciones entre los criterios y subcriterios, que fueron escogidos por la importancia o relación que representaban en la solución del problema, y que consideraban, además, la importancia por sus políticas de calidad, mercadeo, compras, sostenibilidad, etc., para la entidad que requería de la solución de dicho problema.

Después de la escogencia de estos criterios y subcriterios, se procede a hacer una serie de relaciones entre ellos, primero de criterios con criterios, de lo cual resulta una matriz que luego los expertos valoraron, teniendo en cuenta la importancia de los mismos frente al objetivometa, de la misma manera se procede con la valoración de los subcriterios, teniendo en cuenta que se debía comparar entre pares de subcriterios, que correspondían al mismo 42 criterio.
Luego, se pide a los expertos que, de acuerdo a sus conocimientos, procedan a hacer las comparaciones respectivas y dar un juicio, haciendo uso de una escala válida para la toma de decisiones. Con esta información y después de proceder a hacer los análisis respectivos, estos valores se normalizaron, para continuar con el proceso.

Con relación a la valoración de alternativas, se procedió a hacer el análisis de la misma manera que con los criterios y subcriterios, teniendo en cuenta que se debía comparar entre pares de alternativas (en este caso los proveedores por insumo), para cada uno de los subcriterios de último nivel.

Después de determinar estos valores, se procedió a seleccionar el mejor proveedor para cada insumo a partir de la ponderación entre el peso global de los subcriterios y el peso de cada alternativa en los mismos.

Cabe anotar, que el análisis de la información aquí presentada, se hizo gracias al respaldo de una base conceptual de actualidad en el tema de estudio, que soportó la argumentación en cada uno de los pasos de la metodología.

También es importante, aclarar que la información aquí consolidada, presentó de una manera sólida y clara, el resultado de una investigación donde se aplicó la metodología multicriterio en la selección de proveedores, en una empresa del sector azucarero. Buscando siempre, el desarrollo de la misma, de una manera lógica, que llevasé una secuencia de fácil compresión tanto para los expertos y las personas que tienen relación con el abastecimiento en la empresa caso de estudio, como de los lectores de este artículo.

Por último, es importante anotar, que lo realizado en esta investigación en cuanto a la aplicación de la metodología multicriterio y específicamente al método AHP, se hizo de tal manera, que se pudo comprobar, que la teoría funciona de una manera extraordinaria, pues tiene una lógica y secuencia muy clara, de cómo darle solución a un problema multicriterio. Además, se comprueba también, que la participación de expertos en el tema objeto de estudio, es supremamente adecuada y enriquecedora en la solución del problema y que no se necesita, que los expertos conozcan 
previamente la técnica, para poder participar. También se puede decir, que explicar el funcionamiento de esta metodología no es complicado y que su utilización es adecuada y se puede aprovechar en la solución de diversos problemas en las empresas.

\section{Conclusiones}

A partir del desarrollo teórico-práctico de este trabajo se pueden realizar las siguientes conclusiones:

- En la toma de decisiones organizacionales, se debe considerar como criterio principal la sostenibilidad en busca de la competitividad y el desarrollo sostenible. Esto contribuye con el tiempo, a que las organizaciones sean económicamente viables, ambientalmente soportables y socialmente equitativas.

- Las decisiones de selección de proveedores dentro de las compañías son claves para el adecuado funcionamiento de la cadena productiva y deben ser sistémicas, es decir, deben reflejar todos los aspectos que involucran una alta competitividad, así como también identificar todos los actores que deben participar de dichas decisiones.

- Son diversos los métodos y/o mecanismos existentes en la literatura y en la práctica, para tomar este tipo de decisiones. Por lo tanto, es necesario que cada empresa evalúe cual es el mecanismo que mejor se ajusta a su operación y capacidad, para hacer la toma de decisiones más apropiada para la compañía.

- A pesar que existen múltiples métodos que se pueden aplicar para la selección de proveedores, se debe usar, el que mejor se adapte a cada empresa. Para este caso se seleccionó el método AHP, por conocimiento, manejo y practicidad para los expertos que intervinieron en el problema.

- Los criterios y subcriterios elegidos, obedecieron a políticas de calidad y sostenibilidad de la empresa caso de estudio. Luego, las diversas relaciones que se dieron entre ellos y su grado de importancia, tuvo el aporte de unos expertos que fueron escogidos, por el conocimiento y su grado de decisión, en el proceso de compra y abastecimiento de la cadena de abastecimiento.

- Es importante resaltar, que las alternativas relacionadas con la escogencia de proveedores presentadas en esta investigación se dieron por la definición de requerimientos de la empresa caso de estudio, entre los cuales se tuvo: información general del proveedor, ubicación, precio contra competencia, legalidad, derechos humanos, medio ambiente, prácticas laborales, prácticas éticas.

- Con relación a este punto de alternativas, se hizo un análisis de sensibilidad, con otra alternativa (proveedor), de uno de los insumos críticos seleccionados, que cumpliendo en su mayoría todos los requerimientos, menos el de ubicación (cercanía), se podía tener en cuenta en caso, que se tuviese alguna dificultad con el proveedor escogido.

- Con la aplicación de la metodología multicriterio y específicamente del método AHP, se seleccionaron los proveedores que cumplían los requerimientos de la empresa caso de estudio, con relación a los insumos que se priorizaron, de una lista de insumos suministrada.

- Es importante aclarar, que el método empleado en este trabajo puede ser empleado y replicado para la selección de proveedores, de cualquier tipo de insumo de cualquier empresa.

\section{Conflicto de intereses}

Los autores declaran no tener ningún conflicto de intereses.

\section{Referencias bibliográficas}

I. ÁVILA GÓMEZ, Diana Carolina. Cadena de abastecimiento sostenible, iniciativa que contribuye en el crecimiento de las compañías y del país. Gerencia en logística integral. Universidad Militar Nueva Granada: Bogotá. 2014. https://pdfs.semanticscholar. org/0aee/8f I5 |760ce25073837dea87 | |2b85 I92 I dcf.pdf

2. BALLOU, Ronald.Logística.Administración de la cadena de suministro. Quinta edición. Pearson educación. México. 2004.

3. BARRENECHE-GIRALDO, Daniel. Metodología para la evaluación y selección de proveedores. Departamento de ingeniería mecánica. Universidad EAFIT. 2010. https://repository.eafit.edu.co/bitstream/ handle/I0784/425/Daniel_BerrenecheGiraldo_2010.pdf?sequence $=$ I

4. CABELLO-HERCE, Adrián. Métodos de decisión multicriterio y sus aplicaciones. Universidad de la Rioja. Servicio de Publicaciones. Logroño. 2017. https://biblioteca.unirioja.es/tfe_e/TFE002504.pdf

5. CASTILlO MARTíN, Patricia. Política económica: crecimiento económico, desarrollo económico, desarrollo sostenible. En: Revista InternacionaldelMundoEconómicoydelDerecho.volumen III.20I I,p. I 12. http://www.revistainternacionaldelmundoeconomicoydelderecho. net/wp-content/uploads/RIMED-Pol\%C3\%ADticaecon\%C3\%B3mica.pdf

6. CHOPRA, Sunil; MEINDL, Peter. Administración de la cadena de suministros. Estrategia, Planeación y Operaciones. Tercera Edición. 
Pearson educación, México. 2008. https://ulisesmvl.files.wordpress. com/2015/08/administraciondelacadenadesuministrosunilchopra3rae dicion-|505270|232I-Iva I-app689I.pdf

7. FUNDACIÓN IBEROAMERICANA DE ALTOS ESTUDIOS PROFESIONALES. (FIAEP). Control y manejo de inventario y almacén. 2014. https://docplayer.es/420817-Control-y-manejo-de-inventarioy-almacen.html

8. GARCÍA-GÓMEZ, Alejandro. Metodología para la evaluación y selección de proveedores de servicios de mantenimiento. Proyecto de grado. Ingeniería Biomédica. Universidad Autónoma de Occidente. Cali.Mayo,2013,p.30. https://red.uao.edu.co/bitstream/106/4/5130/I/ TBM0I50I.pdf

II. GARCÍA-CASCALES, María Socorro; LAMATA, María Teresa. Selection of a cleaning system for engine maintenance based on the analytic hierarchy process. In: Computers \& Industrial Engineering. Mayo, 2009.Vol. 56, no. 4 p. I442-I 452.

https://doi.org/10.1016/j.cie.2008.09.015

12. GONZÁLEZ-GONZÁLEZ,Aleida; GARZA-RíOS, Rosario.Aplicación de las técnicas multicriteriales en la evaluación y selección de proveedores. En: Revista Ingeniería Industrial. Universidad Tecnológica de La Habana. 2003. vol. 24 No. 2. P. 34-39. http://rii.cujae.edu.cu/ index.php/revistaind/article/view//89//73

13. HANIFAN, Gary; SHARMA, Aditya; MEHTA, Paras. Why a sustainable supply chain is good business? Accenture. In: The Journal of highperformance business. 2012. no. 3. https://www.accenture.com/ t20150522t06161I_w_/ph-en/_acnmedia/accenture/conversionassets/outlook/documents/I/accenture-outlook-why-sustainablesupply-chain-is-good-business.pdf

14. MORALES-FLORES, Francisco Javier.Aplicación de métodos de toma de decisiones multi-atributo en la definición de prioridades en la gestión de infraestructuras en san Luis potosí, México.Tesis Doctoral. Universidad Politécnica de Madrid. Escuela Técnica Superior de Ingenieros de Montes. Madrid. 20II. http://oa.upm.es/9793/I/ FranciscoMorales_TesisDoctoral.pdf

15. RIVEROS BENÍTEZ, Ángela; RODRíGUEZ MELO, Ángela Marcela. Gestión de la cadena de suministro de Bimbo. Misión empresarial. Administración en Logística y Producción. Universidad del Rosario. Bogotá, D.C. 2016. https://repository.urosario.edu.co/ bitstream/handle//0336//2668/RiverosBenitez-angela-2016. pdf? sequence $=$ I \&isAllowed $=y$

16. SAATY, Thomas L. Decision making with the analytic hierarchy process. International Journal Services Sciences, vol. I, no. I, 2008. https://doi.org// 0.1504/IJSSCI.2008.017590

17. SARACHE CASTRO, William Ariel; CASTRILLÓN GÓMEZ, Ómar Danilo; ORTÍZ FRANCO, Luisa Fernanda. Selección de proveedores: una aproximación al estado del arte. Cuadernos de administración. Bogotá. Enero-junio 2009. vol. 22, no. 38. p. 145-167 http://www. scielo.org.co/pdf/cadm/v22n38/v22n38a08.pdf

18. VIDAL HOLGUíN, Carlos Julio. Introducción a la logística y a los sistemas de almacenamiento e inventario. Escuela de ingeniería
19. VIDAL HOLGUíN, Carlos Julio. Fundamentos de control y gestión de inventarios. Escuela de ingeniería industrial y estadística. Editorial Universidad delValle.Cali.2009. https://www.academia.edu/39266025/ FUNDAMENTOS_DE_CONTROL_Y_GESTI\%C3\%93N_DE_ INVENTARIOS

20. VILANA ARTO, José Ramón. La gestión de la cadena de suministro. Nota técnica 2.0I. Dirección de operaciones. Executive MBA. Escuela de organización industrial. 2010-2011. https://es.slideshare.net/ AgenciaExportadora/cadena-de-suministros-44892669

21. VIRSEDA GALLEGO, Laura. Revisión de los métodos, modelos y herramientas existentes para la selección de proveedores. Linköpings Universitet INSTITUTE OF TECHNOLOGY. Junio, 20II. Resumen. https://e-archivo.uc3m.es/bitstream/handle/10016/12130/PFC LauraVirsedaGallego_Resumen.pdf? sequence $=\mid$ \&isAllowed $=y$ 